\title{
Author index to Volume 82
}

\section{Key to abbreviations:}

(BR) Book reviews; (L) Letters to the editors

Abel A 1939

Aceto G 348

Adams S 1650

Adlercreutz H 1107

Agsteribbe E 1474

A'Hern R 568

Ahern R 812

Ahner R 1249

Åhren A-M 1332

Ahrens W 227

Ajayi L 1671

Ajioka Y 1689

Akalın İ 249 (L)

Akechi T 800

Alarcon-Gonzalez P 1145

Alexander F 1353

Alexander FE 1117, 1571

Alhava E 900

Alhava EM 2017

Allal AS 1131

Allalunis-Turner MJ 635

Allan D 74

Allegra CJ 560

Allen DS 1577

Allen-Mersh TG 1004

Alvarez-Mon M 1611 (L)

Ambrosetti P 1131

Amnan K 157

Amo-Takyi BK 1407

Anchisi S 1131

Anderson H 1261

Anderson V 705

Anderson VA 255

Ando Y 497 (L)

Angerson WJ 74

Angius A 553

Ångström T 1332

Aninat-Meyer M 65

Anthony DA 1932

Antoine M 412

Antonopoulos MJ 300

Anttila MA 1983

Aozasa K 1446

Araki K 1892

Ariga N 518

Armatis P 1724

Armstrong AA 1117

Armstrong B 1344

Arnett TR 1459

Aromaa A 1107

Arteta B 953

Artuc M 1453

Asada M 1945

Asakawa S 1801

Asakura H 1689

Asanoma K 459

Ascari E 1254

Ashcroft L 1158
Ashihara T 1327

Asmar L 529

Asosingh K 953

Åström M 1387

Atagi S 418

Atkin SL 1312

Atra A 1396

Aubele M 1204

Auersperg N 1415

Austrian Hereditary Breast and

Ovarian Cancer Group 1249

Auterith A 1249

Autier P 1887

Azzarelli A 1271

Baak JPA 368

Babylon A 399

Badie C 642

Bae MH 385

Bae SK 385

Baguley BC 966

Bailey NP 93

Bak M 339

Balli F 702

Balzi M 178

Banno Y 16

Barachini S 905

Barbarano L 1254

Barnes DM 1145

Barozzi C 865

Barozzi P 702

Barrett JC 459

Barrett-Lee PJ 93

Barroso A 1266

Barshishat M 195

Bartolomei M 295

Bartsch G 39

Bartsch H 763

Baser ME 998 (L)

Bassano L 1732

Bataille V 247 (L)

Battista P 348

Bay BH 1198

Bayerdörffer E 1795

Beale PJ 436

Beard SM 81

Becciolini A 178

Becker M 1844

Beijnen JH 1539

Bell J 1111

Bell JA 1163

Bellefqih 871

Bénard J 871

Benini E 270

Benítez J 1266

Benoy I 1896 (L)

Bergamaschi D 1732

Berger P 85
Bergh J 777

Bergman F 1332

Bergonzi C 1254

Bernaudin J-F 412

Bernstein ED 1611 (L)

Bertram B 629

Betts DR 1239

Bevilacqua G 905

Bianco AR 1772

Bianco T 131

Bibby MC 1925

Bicknell R 161, 749

Bièche I 142

Bieri S 1131

Biesová Z 1808

Bigioni M 480

Bignell G 151

Biomarkers Ad-hoc Group of the

United Kingdom Coordinating

Committee on Cancer

Research 1625, 1627

Birch JM 1571

Black DM 705

Black MH 36

Black MJ 757

Blair V 1571

Blamey RW 1163

Blanco I 535

Blijham GH 1914

Blomqvist CP 777

Boavida M 323

Bocci G 905

Bocciolone L 295

Boddy AV 1519

Bodin L 1387

Bodingbauer Y 1149

Boffetta P 227, 1344

Bogdanova TI 315

Böhles H 399

Bojunga J 1650

Bolton E 167

Bombled J 818

Bonaïti-Pellié C 1939

Bonilla F 1183

Borel Rinkes IHM 1017

Borriello A 1171

Bos AME 1920

Bosch A 151

Bosetti C 204

Boshoff C 702, 812

Bosq J 1614 (L)

Bounacer A 308

Bourhis J 642

Bowrey PF 167

Boxall F 436

Boyle P 1887

Bras-Gonçalves RA 913

Braybrooke JP 1759, 1776
Breitkreutz R 399

Brennan P 1344

Bressac-de Paillerets B 818, 871, 1939

Bribes E 1965

Bridge J 823

Brinton LA 1600

Brock CS 608

Bromsztyk K 1041

Brown KW 323

Brown N 844

Brown R 392

Brugières L 1939

Brugnatelli S 1254

Brunet J 535

Brussen J 1577

Buchs N 858

Buckley CH 760

Buckner J 1371

Budai B 492

Budroni M 553

Bullard J 1111

Bullbrook RD 1577

Bult P 381

Bunch KJ 1339

Bundred NJ 354

Bunnell H 315

Bürkle A 629

Buskens E 1017

Butler LM 131

Butler SA 1553

Büttner B 1844

Buzdar AU 529

Cai J 1317

Caillou B 308

Calvert AH 1519

Cama A 348

Cameron DW 446

Campbell NC 1863

Camplejohn RS 1145

Capella G 535

Caput D 823

Carbone R 1171

Carella R 865

Carelle N 142

Carinci F 1613 (L)

Carls PF 1613 (L)

Carreras J 20

Carss A 315

Carter R 1103

Carter RL 1396

Cartwright RA 1117

Casellas P 1965

Cassidy J 1863

Castelao JE 1364

Casu G 553

Cebrián A 1266 
Cecic I 1835

Cerundolo V 1058

Cha HJ 385

Chakraborty S 1682

Chandra S 1764

Chang J 538

Chang JT-C 1953

Chang S-H 1871

Chaplin DJ 1835

Charles AK 323

Chave HS 124

Chen C-Y 852

Chen HJ 1694

Chen K-W 852

Chen TH-H 1871

Chen Y 2002

Cheng A-J 1953

Cherstvoy ED 315

Chessa L 1945

Cheyns P 1123

Chi Y 657

Chiappetta G 315

Childers SR 1223

Chinje EC 651

Chiron M 642

Choi HS 1403

Chompret A 1939

Christodoulos K 1759

Cianchi F 178

Claeskens A 1123

Clarkson P 1348

Climent F 20

Cline JM 1223

Coates M 1344

Coates RJ 1600

Cocorocchio E 524

Cocquyt V 823

Coderre JA 1764

Cohen B 705

Cohen BB 1247

Cohen Tervaert JW 472

Cole S 1925

Coleman G 568

Coleman MP 1111

Coleman N 1218

Coleman RE 858, 1186, 1547

Colin D 1344

Collan Y 1656

Collette L 283

Collins HM 960

Collins VP 543, 1218

Colombo N 295, 616

Colston KW 1459

Conti E 204

Cook AM 1959

Cookson S 1009

Cools M 1123

Cooper RA 1177

Cooperative Group of Study and

Treatment of Multiple

Myeloma 1254

Cornelisse CJ 151

Corsini C 524

Cossu A 553

Costa-Pereira AP 1827

Cotter TG 1827

Cotterill SJ 1636

Couper R 945

Couturier D 871

Cowled PA 131
Cox G 1427

Cranston DW 161

Craven J 213

Crayford T 742

Cremonesi M 295

Crew J 161

Crognale S 348

Cronauer MV 39

Cucci F 702

Culig Z 39

Cummings J 1629

Cummings MC 1204

Cummins R 1740

Curia MC 348

Curmi PA 142

Curtin NJ 924

Curtis D 330

Cuschieri A 213

Cuzick J 1348

Czajka A 1485

Czarnotta B 1851

Dabrowska A 1485

Daidone MG 270

Dalgleish AG 1009, 1619

Dalibor DV 1757 (L)

Damecour E 263

Damiano V 1772

Danel C 412

Danesi R 905

Danielsen T 1528

Dann JL 705

Dansonka-Mieszkowska A 579

Darnton SJ 1510

Das H 1682

David AS 742

Davidson RH 705

Davidson SE 1177

Davies J 760

Davies MM 1004

Davies SM 753

Davies T 208

de Benedetti A 161

de Boeck G 1000

de Bruijn EA 1000

De Cicco C 295

de Graaf H 1920

De Greef C 953

de Jong FH 112

de Jong JS 368

de Leede G 806

de Leij LFMH 472

De Lena D 560

De Lorenzo S 1772

De Mey R 705

De Mulder PHM 772

De Paepe A 823

De Palo G 270

De Paoli A 1254

de Reijke TM 283

de Schipper FA 1421

De Stavola BL 1577

De Veerman M 953

De Vos F 291

de Vries EGE 767, 1920

de Wilt JHW 973, 1000

de Winkel A 492

De Wolf-Peeters C 823

Dearnaley DP 1959

Declerck PJ 1702
Dedola MF 553

Dejosez M 1851

Del Governatore M 56

del Re EC 1317

Del Tacca M 905

Delfini C 1254

Delia D 1945

Della Ragione F 1171

Della Torre G 1271

Dellian M 1513

Delmas PD 858

Demidchik EP 315

Denis MG 1283

Denison U 1138

Denley H 1163

Depisch D 98

D'Errico A 865

Desai SB 1312

Desjardins L 818

Dessarps-Freichey F 1939

Dettke M 441

Devereux S 278

Devesa SS 1875

Devilee P 151

Dhaene K 1051

Di Biase AR 702

Di Liberti G 1732

Di Paolo A 905

Di Simone M 865

Di Stasi M 1254

Diamandis EP 361

Diel IJ 1381

Dietel M 65, 488

Dieumegard B 871

Dillner J 1332

D’Incalci M 524, 1732

Dinjens WNM 1510

Dirix LY 1896 (L)

Dixon JE 666

Dixon JM 1629

Dixon RM 1776

Dobbs N 1895 (L)

Dobrovic A 131

Dobson LS 1547

Dohjima T 16

Doihara H 46

Dominguez F 584

Dominguez G 1183

Dondon M-G 1939

Dono K 1211

Double JA 1925

Dougal M 1261

Dröge W 399

Du-Villard JA 308

Duddy PM 1145

Dumont JE 315

Dunbar PR 1058

Duncan I 1348

Durany N 20

Dutch Immunotherapy Working Party 772

Dutrillaux B 913

Duval N 263

Eatock MM 1932

Eaton N 1103

Ebert MPA 1795

Ebert W 782

Eden OB 1396, 1571

Edler L 399
Edwards DR 1612 (L)

Eeles R 499 (BR)

Eeles RA 1145

Egashira A 1892

Eggermont AMM 973, 1000

Eguchi H 1211

Eguchi K 104

Eisen T 812

Eiser JR 1605

Ekert H 255

Ekström C 1561

Ekström TJ 1561

El-Abassi M 1932

Elbe B 1844

Elias E 742

Elliott AM 1863

Elliott P 1103

Ellis IO 1163

Elstner E 452

Engers R 1063

Enomoto T 1446

EORTC Genitourinary Group 283

Eppinga P 806

Epstein J 1433

Erba E 1732

Erdkamp FL 1914

Eriksson T 1561

Erjavec Z 806

Erselcan T 777

Esiri MM 1441

Eskelinen M 900

Eskelinen MJ 2017

España P 1183

Esposito DL 348

Esslimani M 1965

Ethier SP 666

Etienne MC 171

Ettore F 171

Evans DGR 998 (L)

Evans H 608

Evans MF 424

Evans TRJ 1932

Ezaki T 1814

Fabbro D 1063

Fabbrocini A 1772

Fader DJ 657

Faircloth G 1732

Fallowfield L 1783

Fan Z 1993

Fang M 1993

Faragher EB 354

Faraoni P 178

Farmery SM 1233

Farris A 553

Fayers PM 213

Fazio F 616

Fears TR 1875

Fehringer M 794

Fei G 1795

Feifel G 794

Felberbauer FX 1758 (L)

Feleszko W 1485

Fentiman IS 1577

Fenton BM 937

Ferguson-Smith MA 1218

Fernández PL 20

Feroze F 538

Ferrero JM 171 
Ferrucci PF 524

Ferruzzi L 865

Feunteun J 1939

Ficari F 348

Fichtner I 1844

Fielding J 213

Fiorentino M 865

Fiorenza M 295

Fleischmann E 1249

Fogli S 905

Fokkema E 767

Fong KM 1191

Fontanella E 1945

Fordyce A 705

Formento JL 171

Fosså SD 737

Foulkes WD 757, 1646

Fox SB 844

Franceschi S 204, 1860

Francoual M 171

Franko AJ 635

Frati L 1945

Fraumeni Jr JF 718, 1875

Frayling IM 827

Frébourg T 1939

Freedman LS 213

Freeland J 1117

Frelinger JG 937

Freshney RI 881

Friedmann A 1249

Friery OP 1469

Frigerio L 616

Frisch RE 726

Frout D 263

Fuggle S 161

Fujii A 404

Fukuda J 1415

Furuse K 418

Fusco A 315

Fusenig NE 591

Gabbert HE 1063, 1851

Gaborieau V 227

Gago-Dominguez M 1364

Galanis E 1371

Galettis P 966

Gallagher CJ 2024

Gallagher R 1469

Galligan ES 1469

Gallinger S 1646

Gallus S 1860

Gamallo C 1183

Gambini C 1171

Gammon MD 1600

Ganesan TS 1759, 1776

Ganly I 392

Garbe C 1149

Garcia JM 1183

Gardes M 1939

Garnero P 858

Garnham J 330

Gatter K 1427

Gatter KC 844, 1441

Gebhardt MC 1327

Gee JMW 501

Geerts ML 823

Gellert K 65

George L 731

Geradts J 1191

Gerharz CD 1063, 1851
Gerrard M 1396

Gerth R 85

Gertsch P 1131

Ghadirian P 757, 1646

Ghielmini M 524

Ghilchik MW 492

Giannoudis A 424

Giatromanolaki A 844, 1427

Giermasz A 1485

Gilchrist R 1145

Giles GG 1887

Gillespie AM 1186, 1393

Giordani L 1171

Giordano M 1254

Godard V 412

Godber T 255

Goepel JR 1186

Gokhale DA 1117

Gołąb J 1485

Goldstone AH 278

Gonthier M-P 142

González I 535

Gonzalez R 1183

González-Aguilera JJ 535

Gore ME 812

Gorin I 818

Gough AC 124

Gourgou S 1965

Grabau DA 339

Grabbe J 1453

Graf AH 1138

Graham DI 74

Grana C 295, 616

Grandjouan S 871

Gray IC 1671

Gray NE 1671

Gray SG 1561

Greenberg RS 718

Gregory RK 1907

Grenier J 1965

Griffin MJ 1519

Griffiths JR 2009

Grigioni WF 865

Groen HJM 767, 806

Groenendijk RPR 381

Groenewegen G 772

Grohs JG 85

Groot K 1724

Gryboś M 621

Guerry M 1614 (L)

Guillou PJ 1233

Gullick WJ 1163

Güllü IH 249 (L)

Günther K 1407

Gupta RK 2024

Gustafson TA 683

Gusterson B 568

Habib FK 186

Hack V 399

Haddada H 642

Hagmüller E 399

Hahn L-J 1871

Haider K 98

Hallmans G 1332

Halm U 1013

Halmos G 1724

Hambley TW 966

Hamblin MR 56

Hamburger AW 683
Hamel N 757, 1646

Hamidi S 1433

Hamilton JA 1671

Han C 1759

Hancock BW 1186, 1393, 1547

Handt S 1407

Hann IM 1396

Hansen S 339

Hara A 467

Harada M 104

Harding C 354

Harland C 1605

Harper P 1158

Harris AL 161, 651, 844, 1427, 1441, 1759, 1776, 1895 (L)

Hartmann O 251

Hartschuh W 591

Hasan T 56

Hasegawa K 1682

Hashiguchi S 1327

Hattori Y 657

Hausmaninger H 1138

Hayashi S 838

Hayashi Y 1801

Hayes RB 718

Hayward JL 1577

Hebert F 1724

Heinrich J 227

Heinzer H 246 (L)

Helbich T 1249

Helfrich W 472

Heliövaara M 1107

Helmbach H 488

Helzlsouer KJ 731

Henderson BE 1867

Hendrich S 1879

Hennig E 1041

Henriksson R 246 (L)

Henry-Amar M 263

Henz BM 1453

Heo SC 1403

Herfarth C 157

Heriot AG 1009

Hernberg M 777

Herrington CS 424

Hibi T 1717, 1814

Higashi T 833

Higashiyama M 374

Hill C 1614 (L)

Hillen HF 1914

Hipfel R 1149

Hirabayashi K 981

Hiraki S 104

Hiraki Y 104

Hirasawa Y 1327

Hirata M 1327

Hirst DG 1469

Ho L 1348

Ho S 497 (L)

Hobisch A 39

Hobson R 1396

Hoekman K 772

Höfler H 315, 1204

Högel J 118

Holdaway IM 241

Hollstein M 763

Holm E 399

Holm S 1561

Hölting T 157

Holzer G 85
Hombauer H 1290

Honma T 1689

Hoodfar E 705

Hooper ML 1247

Hoover RN 718, 1600

Hopewell JW 1764

Horii A 1801

Horiuchi S 891

Horsburgh T 186

Hortobagyi G 529

Horwich A 1959

Hosaka T 1677

Hoshi M 1801

Hosoe S 418

Houlston RS 568

Howard GCW 136

Howarth GS 945

Howe FA 2009

Howell A 354

Howell SB 34

Howell SJ 789

Hsieh T-J 1035

Hsu C-H 1035

Hsu C-P 1480

Hsueh S-F 1480

Hua D 1939

Huang C-I 374

Huang TH-M 514

Huckriede A 1474

Huddart RA 1959

Hudis C 1897

Hughes AN 1519

Hughes CM 1469

Hughes-Fulford M 2002

Huland E 246 (L)

Hunter RD 1177

Hutzler P 1204

Ichikawa T 838

Ichimura K 543

Ichimura T 1682

Ikeda Y 1892

Ikram MS 1553

Iles RK 1553

Imamura M 429

Imeson JD 1396

Ings SJ 278

Ingvar C 1593

Innocenti F 905

Inoue $\mathrm{T} 675$

International Bone and Cancer

Study Group 1381

Iolascon A 1171

Irrissuto C 480

Ishii H 1717,1814

Isonishi S 34

Ito I 1415

Ito Y 1211

Izzo A 1171

Jackler RK 998 (L)

Jackson RT 241

Jacobs MV 1421

Jain RK 1513

Jaiswal AK 1305

Jakóbisiak M 1485

James WH 2025 (L)

Janeczko M 550

Janicot M 642

Janik P 1041 
Janot F 642, 1614 (L)

Jansen CT 248 (L)

Jansen RLH 772

Jarrett RF 1117

Jay G 1959

Jayasurya A 1198

Jayne DG 1233

Jayson GC 760

Jemal A 1875

Jenkins V 1783

Jeong S-Y 1403

Ježek J 1808

Jiang W 118

Jiménez E 1662

Jimenez OM 20

Jimeno J 1732

Jöckel KH 227

Joensuu H 777

Johansson R 1332

Johnsen R 1358

Johnson TM 657

Johnston A 1519

Johnston PG 560

Johnston SRD 812

Joly F 263

Jonas SK 1004

Jonges TGN 931

Jonker DJ 1789

Jonsson N 1593

Joseph J 20

Joseph P 1305

Jowitt SN 1261

Jung K 1611 (L)

Jung M 1611 (L)

Jung PM 1403

Jung S-M 1953

Kaganoi J 429

Kaghad M 823

Kahlos K 1022

Kaijen P 1123

Kaina B 629

Kainulainen T 1433

Kainz C 1138

Kairemo KJA 777

Kaisary AV 1671

Kakolyris S 844

Kalinski T 1851

Kalipciyan M 1276

Kallincos NC 945

Kambouchner M 412

Kameoka S 404

Kamiński R 1485

Kamradt MC 1709

Kanai T 1717, 1814

Kanazawa M 518

Kane E 1117

Kaneko C 518

Kaneko Y 1801

Kaneyoshi T 833

Kanno H 1446

Kano M 429

Kaprio J 248 (L)

Karabon L 621

Kariyama K 833

Karjalainen JM 2017

Karner J 98

Karner-Hanusch J 1276

Karpińska G 579

Kasajima T 404
Kashiwagi K 1814

Kato H 459, 981

Kato K 891

Kato T 404

Katsikas M 300

Kaur S 1004

Kawabata K 467

Kawaguchi H 1892

Kawaguchi T 418

Kawahara M 418

Kawahara S 104

Kaye SB 392

Ke YQ 1694

Keilholz U 118

Keim V 1013

Keizer HJ 1539

Kelland LR 436

Keller G 315

Kellokoski JK 1983, 2017

Kelsey S 2024

Keng PC 937, 1223

Kerin MJ 1312

Kerr B 1519

Kersey JH 234

Kew TY 1163

Kim JC 1403

Kim KW 385

Kim NK 1403

Kimura T 404

King J 167

King M-C 705

Kinlen LJ 999

Kinnula VL 1022

Kinoshita Y 88

Kirby JA 1900

Kirkwood JM 1755 (L)

Kitamura K 1892

Kitchener HC 760

Kiura K 104

Kjellberg L 1332

Klar E 157

Klijn JGM 151

Klimczak A 621

Klocker H 39

Knekt P 731, 1107

Kobayashi M 838, 953, $1689 \quad$ La Vecchia C 204, 1860

Kobayashi Y 833, 838

Koch CJ 937

Kocha W 1789

Koeffler HP 452

Koehler A 931

Koepsell H 629

Kogner P 1561

Kohno N 374

Kohshi K 88

Koizumi T 1682

Kolonel LN 1867

Kondo H 459

Konopa J 1300

Konopiński R 579

Koomägi R 1747

Koot VCM 1017

Korbelik M 1835

Kornek GV 98

Kos J 782

Koshiuka K 452

Koskenvuo M 248 (L)

Kosma V-M 1983

Kosma VM 2017

Kosmas C 300

Krašovec M 782

Kunugita N 88
Kotz R 85

Koukourakis M 844

Koukourakis MI 1427

Kowalczyk P 1041

Kozar K 1485

Kramar A 1965

Krammer P 1851

Kraszewska E 579

Krauss G 98

Kravdal Ø 737

Kreienbrock L 227

Kreuzer M 227

Kroesen BJ 472

Kronqvist P 1656

Krueger E 1709

Kruit WHJ 772

Kryczek I 621

$\mathrm{Ku}$ J-L 1403

Kujala HE 2017

Kumar A 960

Kumar D 1009

Kumar S 1393

Kunisue H 46

Kunkler IH 1247

Kuopio T 1656

Kuppen PJK 1539

Kupryjańczyk J 579

Kurdoğlu M 249 (L)

Kurebayashi J 46

Kurek R 1650

Kuriaki Y 891

Kurian KM 1247

Kurosumi M 46

Kurttila E 1022

Kurtz JM 1131

Kusterer K 1650

Kusumoto M 1819

Kusuzaki K 1327

Kutoh E 1123

Kwaan HC 1702

Kwasny W 98

Kwon YG 385

Lacava J 560

Lacave R 412

Lachkar S 142

Lacroix J 157

Lage H 488

Lah TT 782

Lahodny J 1138

Lahousen M 1030

Lakhani SR 568

Lal G 1646

Lalisang RI 1914

Lambert J 1051

Lang SH 990

Langbauer G 1249

Lange A 621

Larjava H 1433

Lasek W 1485

Lasser P 871

Late Effects Group of the United Kingdom Children's Cancer Study Group 1636

Lau WY 497 (L)

Laurent-Puig P 913

Laux DE 514
Lavail R 1965

Lawrence D 1117

Le Bihan M-L 871

Leake R 1625, 1627

Lebailly P 263

Leblanc-Talent P 171

Lee C 1519

Lee $\mathrm{H} 852$

Lee H-S 852

Lee J 937

Lee $\mathrm{OH} 385$

Lee SM 1158

Lee YM 385

Leek RD 844

Lefrère I 871

Lehnert M 538

Leigh IM 844

Leitz W 220

Lemerle J 1939

Lemoine NR 1058

Lendeckel U 1795

Leonard JH 823

Leone B 560

Leone G 1610 (L)

Lequeux N 1965

Leridant AM 1614 (L)

Lerner K 1433

Lessor T 683

Leung PCK 1415

Leung WT 497 (L)

Leverett D 278

Levesque MA 361

Levi F 204

Levitt NC 1759, 1776

Lévy C 818

Lewis IJ 1339

Li Z 429

Liang B 1519

Liao C-T 1953

Libroia A 1772

Lidereau R 142

Liede A 705

Liff J 718

Ligot L 1939

Lim S 538

Lin P 852

Lin S-R 1035

Linch DC 278

Lindh J 601

Lindhorst E 1650

Linet MS 234

Lister TA 2024

Little J 1863

Littlewood TJ 1776

Liu B 1993

Liu G 1646

Livant DL 666

Liyim D 1186

Lluis F 535

Lobo F 1266

Locker G 98

Loening SA 1611 (L)

Logue JP 1177

Loibner H 441

Loidi L 584

Loimas S 900

Lomax ME 1145

Lombardi A 348

Lopes A 1348

Lord EM 937 
Lorenz I 1851

Lorigan P 1158

Lorigan PC 81, 1547

Lu Y 1993

Luboinski B 1614 (L)

Luisi A 270

Lund Nilsen TI 1358

Luppi M 702

Lustenberger P 1283

Lutz J-M 1111

Lykkesfeldt AE 1844

MacCallum J 1629

McCaw DL 1297

McCormick C 881

MacGregor A 247 (L)

Mach RH 1223

Machaj E 1485

Machiavelli M 560

Machida D 571

McIntyre IA 1469

MacKay EH 186

McKeage MJ 966

McKean-Cowdin R 1867

McKenna SL 1827

McKenzie K 742

MacKenzie MAF 1247

McKeown SR 1469, 1925

McKinna A 568

McMichael AJ 1058

MacMillan A 278

McNally RJQ 1571

McRae CU 241

Mader RM 1276

Maehama T 666

Maehara N 1819

Mafune K-i 1557

Magarey C 167

Magdalena C 584

Magener A 157

Maggi CA 480

Maggioni A 295

Magklara A 361

Magnani P 616

Mahotka C 1851

Mairs RJ 74

Maitland NJ 990

Mak I 812

Mäkinen K 900

Malamos NA 300

Malfertheiner P 1795

Mali WPThM 1017

Malik KTA 323

Mallone S 227

Malvy D 263

Maly K 39

Mamelle G 1614 (L)

Mancuso P 524

Mandyam R 635

Mangili G 616

Mangioni C 295

Mankin HJ 1327

Mann JR 1568

Mannermaa AJ 2017

Mansi JL 1459

Manzini S 480

Marchetti A 905

Marcuello E 535

Marczell A 98

Marenbach K 1662
Mariani CDA 616

Mariani-Costantini R 348

Marinelli A 1539

Markwart SM 666

Marossy A 568

Maroun JA 1789

Marr P 167

Marras V 553

Marriott JB 1009

Marshman E 924

Martelli G 270

Marth C 1138

Martinelli G 524

Martínez B 1266

Martínez N 1662

Marx D 1662

Masaki T 9

Måsbäck A 1593

Massarelli G 553

Masson D 1283

Massoud C 263

Matano E 1772

Mathieu S 1553

Matsuda T 9, 459

Matsuda Y 1689

Matsunaga K 467

Matsuura N 1211

Matthews J 608

Mattioli S 865

Mattis A 1204

Mattsson A 220

Mazumdar M 550

Meade-Tollin LC 931

Meden H 1662

Mehle C 601

Meijer CJLM 1421

Meijer DKF 472

Meijers-Heijboer H 151

Melbye M 1070

Melia J 1605

Mellemkjær L 1353

Mendelsohn J 1993

Mendez E 1568

Menger MD 794

Merletti F 227

Mertens AC 234

Messerini L 348

Meyer T 1535

Mezzelani A 1271

Mezzetti M 524

Micca PL 1764

Micek M 705

Middleton MR 1158, 1755 (L)

Middleton SB 827

Miehlke S 1795

Milan T 248 (L)

Milano G 171

Miller WR 136, 1629

Minegishi T 1415

Miner C 1145

Minguell JJ 1290

Minna JD 1191

Mitchell PJ 1983, 2017

Mitsui Y 1974

Mittermayer C 1407

Miwa H 1446

Miyakawa A 543

Miyakawa R 404

Miyake M 374

Miyakoshi J 28
Mizumoto K 1819

Mizutani S 1945

Mohideen N 1709

Moisio KI 1983, 2017

Molema G 472

Monaghan J 1348

Monden M 1211

Moon EJ 385

Mooney R 1740

Mora O 1254

Moran JP 937

Mori A 524

Mori H 467

Mori M 1557

Morita M 1892

Moriya T 518

Morris AG 1510

Morris DL 167

Morris GM 1764

Morrison E 1233

Morrison GH 1764

Moscatello DK 186

Möslinger R 1249

Moss S 1605

Mössner J 1013

Mothersill C 1740

Mrzyk S 1063

Muche JM 1611 (L)

Mueller K-M 763

Mukai M 1814

Mulder GJ 1539

Müller M 1851

Müller-Holzner E 1138

Munakata S 1446

Munday GR 1381

Muradore I 1732

Murakami H 1677

Murata H 1327

Murnane MJ 1317

Murphy PA 1879

Murray MM 1469

Muto T 9

Mutton K 1261

Nabers J 806

Naeyaert JM 823, 1051

Nagano H 1211

Nagashima S 981

Nagesh K 1261

Nagy Z 1441

Naka N 418

Nakahara M 1211

Nakamata T 1677

Nakamori S 1211

Nakamura M 1211

Nakamura T 1677

Nakao K 1211

Nakatsukasa H 833

Nakayama T 1677

Nakazawa A 1717

Nambooze S 1585

Namer M 171

Nanus DM 550

Nardelli F 480

Nardini D 905

Narisawa R 1689

Narod SA 705, 1646

Nash A 568

Naundorf H 1844

Neefs JM 1123
Neglia JP 234

Negri E 204, 1860

Nekulova M 1757 (L)

Neshasteh-Riz A 74

Nessler-Menardi C 39

Neumann C 1844

Newell DR 924, 1519

Newland AC 2024

Newlands ES 608

Newton CJ 1312

Nicholson RI 501, 1163

Nicoletti G 1254

Niggli FK 1239

Nilsson I 1387

Nishida J 891

Nishikawa T 404

Nishimura R 1682

Nishio S 1819

Nishiwaki Y 800

Noguès C 142

Norman A 1959

Norrback K-F 601

Norrish AE 241

Nortier JW 1914

Nosarti C 742

Nose T 1974

Nostro MC 178

Nouso K 833

Nozawa Y 16

Nuutinen P 900

Obiezu C 361

O'Brien MER 93

O'Byrne K 1759, 1776

O’Byrne KJ 1427, 1895 (L)

O'Doherty CA 2024

O'Dwyer ST 7

Oefner P 1249

Oehler MK 749

O'Flaherty E 1900

Ogawa T 1819

Ogawara M 418

Ogg GS 1058

Ohi R 1801

Ohkawa K 34

Ohno S 1557, 1892

Ohno T 16

Oka M 1677

Okayama Lung Cancer Study

Group 104

Okayasu I 571

Okishio K 418

Okui T 838

Okuyama T 800

Olapade-Olaopa EO 186

Ołdak T 1485

Olive PL 635

Oliver RTD 2024

Olmeo N 553

Olopade OI 705

Olsen JH 1353

Olsson H 1593

Ongenae N 1123

Onishi T 833

Oomen MH 112

Oppenheim D 25

O'Reilly SM 608

Osanto S 772

Osman S 608

Osorio A 1266 
Ossu Rocca PC 553

Ostrowski J 1041

Osundeko O 354

Otagiri N 1801

Otsuki T 46

Otsuki Y 1446

Owen J 1158

Owens J 74

Pääkkö P 1022

Paganelli G 295, 616

Palicio M 535

Palma C 480

Palmer BD 966

Palmer K 124

Palmieri G 553

Palmirotta R 348

Palomba G 553

Panigone S 1945

Panou C 52

Paolucci M 524

Papandreou CN 550

Pappas TN 691

Paradis A-J 1646

Paradiso A 560

Parish D 492

Park J-G 1403

Park YJ 1403

Parkes SE 1568

Parkin DM 1585

Parkins CS 1835

Parliament MB 635

Parma J 315

Parsons MA 330

Pasca A 553

Pastorek J 1808

Patel H 1764

Patterson AV 651

Patterson LH 1469, 1925

Paul J 1932

Payne JT 1297

Pecorelli S 616

Peelen T 151

Peer PGM 381

Peeters PHM 1017

Peinado MA 535

Pelgrims J 291

Pelloni A 1131

Pendry L 1605

Perez ML 1740

Perone P 657

Perry MR 514

Perry SL 1233

Persico I 553

Petersen I 65

Petersen S 65

Peto J 1111

Petroni S 560

Pettit SJ 1900

Peyrottes I 171

Phillips HA 136

Phillips RKS 827

Piccinini EE 56

Piccinini L 1254

Pierotti MA 1271, 1945

Piersma H 1920

Pietsch T 1561

Pignon J-P 871

Pijl MEJ 1539

Pike MC 1867, 1879
Pilkington GJ 52

Pilotti S 1271

Pilz L 591

Pinder SE 1163

Pinkerton CR 1396

Pirastu M 553

Pires S 323

Pirianov G 1459

Pisano A 553

Pisano M 553

Pistorius G 794

Pittermann E 1249

Pivot X 529

Pohl J 629

Poirée B 263

Pol RP 1421

Polak-Charcon S 195

Pollán M 1662

Pollock AM 712

Polyzos A 300

Pope ER 1297

Pope V 718

Potten CS 178

Potter AM 330

Potter JD 234

Pottern LM 718

Poupon M-F 913

Powell JE 1568

Powlesland RM 323

Pozcharskaya V 315

Preston SR 124

Price PM 608

Primrose JN 124

Probst-Hensch NM 1867

Proebstle TM 118

Propper DJ 1759, 1776

Prové A 291

Provencio M 1183

Prudente S 1945

Puente JL 584

Purdie D 1204

Purohit A 492

Pyle L 812

Rabkin C 718

Radford JA 789

Radloff GA 753

Rahman N 538, 568

Rajagopalan B 1776

Ramp U 1851

Rampling R 74

Read LC 945

Rebhandl W 1758 (L)

Reed MJ 492, 1577

Rees RC 330

Reeves JR 74

Regauer S 1030

Reich O 1030

Rembiszewska A 579

Remes K 601

Renehan AG 7

Renneberg H 1650

Rennie IG 330

Renwick A 705

Reyes E 1611 (L)

Reynolds K 760

Reynolds PA 323

Reza MS 74

Rezvani M 1764

Riccardi A 1254
Ricci S 412

Ridwelski K 1795

Riggi M 524

Rinaldi E 1254

Rishi AK 683

Rissanen H 1107

Ritchie LD 1863

Rivera F 20

Rizzoli R 858

Roberge C 263

Roberts JV 742

Roberts KG 1671

Robertson C 1887

Robertson JD 1261

Robertson LM 74

Robinson D 1111

Robinson SP 2009

Robison LL 234

Robledo M 1266

Röddiger S 1650

Rodriguez FJ 514

Roesch F 227

Rofstad EK 1528

Rogers P 436

Romero A 560

Ronsin M 1939

Ronzoni S 1732

Rooprai HK 52

Roos G 601

Rose C 339

Rosenquist R 601

Ross JA 753

Ross RK 1364

Rostagno P 171

Rosty C 913

Rougier P 871

Rubenstein A 998 (L)

Rucklidge GJ 52

Ruers TJM 381

Rumi C 1610 (L)

Runsink AP 1421

Ruparelia K 1925

Rusin M 579

Rustin GJS 1535

Rutella S 1610 (L)

Rutqvist LE 220

Rybczyńska J 1485

Ryder WDJ 760

Saarikoski S 1983

Sabin C 702

Sabourin J-C 871

Saegusa M 571

Saeki H 1892

Sage RD 446

Said JW 452

Sakae K 104

Sakon M 1211

Sakurai T 429

Salgado R 1896 (L)

Salo T 1433

Sampson FC 81

San Román JM 1266

Sandhu DPS 186

Sandstedt B 1561

Sanoudou D 1218

Santoro M 315

Santos C 635

Sapunar F 812

Sarasin A 308
Sarobba MG 553

Sasaki S 9

Sasano H 518

Sato F 429

Sato N 1819

Saunders MP 651

Sauter ER 361

Savage P 1058

Saviozzi S 1945

Scarffe JH 1261

Schadendorf D 488, 1453

Schäfer BW 1239

Schally AV 1724

Scheithauer W 98

Schiefke I 1013

Schittek B 1149

Schlienger P 818

Schlumberger M 308

Schlüns K 65

Schmidt EE 543

Schmidt M 1993

Schmidt T 657

Schneider J 1662

Schoenberg JB 718

Scholefield JH 960

Scholl FA 1239

Schouten HC 1914

Schratter-Sehn A 98

Schrijvers D 291

Schröder FH 283

Schüder G 794

Schumann T 1013

Schumm-Draeger PM 1650

Schuster K 399

Schwartz AG 718

Schwartz B 195

Schwartz P 167

Schweiger A 782

Sciot R 823

Screnci D 966

Seal S 568

Sedivy R 1276

See L-C 1953

Segawa Y 104

Seifert M 1249

Seker H 629

Selvan RS 691

Senaratne SG 1459

Sengupta PS 760

Severi G 1887

Severson RK 234

Seymour CB 1740

Seymour K 1900

Seynhaeve ALB 973

Shaddick G 1103

Shalet SM 789

Shamash J 2024

Shanks JH 760

Sharp L 1863

Sharp SY 436

Shen R 550

Sheridan MT 1177

Shibuya H 1835

Shimada Y 429

Shimizu K 16

Shimizu N 46, 1801

Shinozaki H 1415

Shiu M-N 1871

Shiwaku HO 1801

Shu XO 234 
Shuja S 1317

Sica S 1610 (L)

Sideri M 295

Silva JM 1183

Silvestrini R 270

Simonato L 227

Simone G 560

Simony-Lafontaine J 1965

Sisley K 330

Skiner R 1636

Skladanowski A 1300

Slade RJ 760

Sleijfer DTh 806

Smedsrod B 953

Smeets JBE 806

Smets EMA 789

Smibert E 255

Smith DR 1764

Smith IE 812, 1907

Smith M 1932

Smith PG 924

Smith RE 931

Snary D 1671

Sng J-H 538

Snieder H 247 (L)

Snijders PJF 1421

Sobczak-Thépot J 642

Sobel A 142

Sodha N 1145

Soini Y 1022

Songini C 616

Sonoo H 46

Sørensen FB 339

Soufir N 818

Soukop M 1932

Soulié P 913

Soutar DS 392

Southern SA 424

Sozzi G 1271

Spanedda R 1254

Spataro V 1131

Spector T 247 (L)

Speirs V 1312

Speleman F 823

Spence HJ 1615

Spierings DCJ 1474

Spinelli R 767

Spitz L 250 (BR)

Sprangers MAG 1131

Springer E 1063

Srinivasan R 1163

Staib A 782

Stamato TD 1740

Stanczyk F 1879

Stanczyk FZ 1867

Standiford SB 514

Stanisch M 1650

Stapleton G 1615

Steel CM 705, 1247

Steger GG 1276

Stelmachów J 579

Stevens MCG 1636

Steward LMD 1671

Steward WP 1427

Stewart AFR 446

Stewart THM 446

Stiller CA 1339

Stockton D 208

Stokłosa T 1485

Stolte M 1795
Stone JG 568

Stoppa-Lyonnet D 818

Stower M 990

Stratford IJ 651, 1177, 1925

Stratford MRL 1835

Stratton M 151

Stratton MR 568

Strickler H 718

Strieth S 591

Stubbs M 2009

Stummvoll W 1138

Suárez HG 308

Sugie S 467

Sugimachi K 1557, 1892

Sugimoto T 1682

Suginoshita T 1327

Sujansky E 998 (L)

Suminami Y 981

Sutherland DJA 361

Suzuki T 518

Suzuki Y 1689

Svoboda K 1702

Swanson GM 718

Sweetenham JW 4

Syrjänen K 1983

Szamel I 492

Szende B 1724

Szepeshazi K 1724

Szymańska G 579

Tabata M 104

Tada Y 452

Tagliabue E 1945

Tagliaferri P 1772

Takano S 1974

Takeda T 1211

Takeshita H 1327

Taki T 374

Talamini R 1860

Talbot DC 1759, 1776

Talbot M 1614 (L)

Tamborini E 1271

Tan NG 1198

Tan W 538

Tanaka K 46, 800

Tanaka M 1819

Tanaka S 1557

Tanaka T 34

Tanda F 553

Tang CK 46

Tanimoto K 838

Tate D 1297

Tavani A 1860

Taya Y 1945

Taylor GM 1117, 1571

Taylor M 844

Tealdo F 524

Teissier E 171

ten Hagen TLM 973, 1000

Tenhunen M 777

Teppo L 248 (L), 1107

Terao Y 891

Terry G 1348

Terry TR 186

Tetlow L 354

Tewarie L 381

Thatcher N 1158

Thomas BS 1577

Thomas GA 315

Thompson CH 1776
Tidefelt U 1387

Tilanus HW 1510

Tilanus MGJ 1510

Timmerman MA 112

Timorek A 579

Tindall DJ 361

Tinelli C 1254

Tingby O 1218

Toguchida J 1677

Toh CH 1694

Tokuchi Y 838

Tokus M 399

Tollenaar RAEM 1539

Tomasic G 270

Tomei F 204

Tomono Y 1974

Tompson RV 1297

Tonelli F 348

Tonini GP 1171

Tonnachera M 315

Tookman A 1485 (BR)

Torchilin VP 1513

Torelli G 702

Tórtola S 535

Tosaki T 88

Toshikuni N 833

Tran M 263

Treweeke AT 1694

Trieb K 85

Tronko ND 315

Trubetskoy VS 1513

Trzeciak L 1041

Tsai J-H 1035

Tsai K-J 852

Tsavaris NB 300

Tsuboi K 1974

Tsuboyama T 1677

Tsuchiya E 838

Tsuda H 675

Tsuji T 833

Tsujimoto M 1211, 1446

Tuomilehto J 731

Turk V 782

Turley H 1427

Turner HE 1441

Tursz T 642

Uchitomi Y 800

Uchiyama I 675

Ueno H 891

Ueoka H 104

Ueoka Y 891

Uges DRA 767

Ugolini G 56

UKCCR Elderly Cancer Patients in Clinical Trials Working Group 1

Umesaki N 675

Umeshita K 1211

Umetani N 9

United Kingdom Childhood

Cancer Study Investigators 1073

United Kingdom Children

Cancer Study Group

(UKCCSG) 1396

Urashima T 1819

Urban T 412

Urdl W 1030

Usadel KH 1650
Uscinska B 213

Usmani BA 550

Vabre L 1614 (L)

Vach W 339

Vahrmeijer AL 1539

Valanzano R 348

Valentini D 1254

Valero V 529

Vallejo C 560

Valota O 767

Van Belle S 823

van Bockel JH 1539

Van Camp B 953

van de Velde CJH 1539

Van den Brande J 291

van der Kwast TH 112

van der Pool S 538

van der Sluis RF 381

van der Veen AH 973

van Dierendonck JH 1539

van Diest PJ 368

Van Gele M 823

van Herpen CML 772

Van Marck E 1051

Van Noorden CJF 931

van Ommen G-JB 151

van Oosterom AT 767

van Putten JWG 806

Van Riet I 953

Van Roy N 823

van Schaik RHN 112

van Steenbrugge GJ 112

van Tiel ST 973, 1000

van Vliet M 151

van Vroonhoven ThJMV 1017

van Weerden WM 112

Vancoillie G 1051

Vande Broek I 953

Vanderkerken K 953

Varani J 657

Varga JL 1724

Varmeh-Ziaie S 543

Vasen HFA 151

Vassal G 642

Vatten LJ 1358

Vaughan ATM 1709

Vaughan MM 812

Vecchio G 315

Vegazo IS 1183

Velek J 1808

Veneroni S 270

Verkasalo PK 248 (L)

Verkooijen HM 1017

Vermeulen PB 1896 (L)

Vermorken JB 291

Verweij J 767

Vesprini D 1646

Vickers N 712

Vignati S 1732

Voest EE 1914

Voit C 118

Vollmar B 794

Volm M 1662, 1747

von Knebel Doeberitz M 157

von Schweinitz D 1561

Voorhorst FJ 1421

Voscoboinik L 315

Vujanovic NL 981 
Wabinga HR 1585

Wabwire-Mangen F 1585

Wadell G 1332

Wager E 93

Wagner TMU 1249

Wake N 459, 891

Walboomers JMM 1421

Wald NJ 731

Wallen CA 1223

Wals J 1914

Walter S 1709

Walton DS 1312

Wan P 1879

Wang DY 492, 1577

Wang H-J 852

Wang J 1702

Wang J-Y 1035

Wang L-M 1223

Wang S-L 852

Wang T-CV 1953

Wang XW 683

Ward A 568

Ward FE 691

Warner E 705

Wạsik M 1485

Wass JAH 1441

Watanabe G 429

Watanabe K 459

Watanabe M 1717, 1814

Watanabe T 9

Watson GJ 1671

Watson SA 960

Watt HC 731

Watts MJ 278

Weber B 705

Weber L 118
Weber T 157

Wegener K 763

Weiskop S 255

Weiss RA 702

Weissgerber G 1063

Weitz J 157

Welker P 1453

Wen W 234

Wen-yi Y 16

Wenzel M 1851

Werle B 782

Werner M 1204

Wersäll P 246 (L)

Wesch H 763

West CML 1177

West DC 1694

West MK 1297

Westerdahl J 1593

Weytjens R 1896 (L)

Whateley TL 74

Wheeler KT 1223

Whelan P 283

Whitby D 702

Whiteside TL 981

Whitley E 227

Wicker R 308

Wideman CL 74

Wierikx CDJ 112

Wießler M 629

Wijnhoven BPL 1510

Wiklund TA 777

Wilczyński G 1485

Willemse PHB 1920

Willemsen EL 492

Williams ED 315

Williams GH 315
Williamson E 452

Wils JA 1914

Wilschut J 1474

Wilson JW 178

Wilson P 1932

Windbichler GH 1138

Winter R 1030

Witzigmann H 1013

Wobbes T 381

Wocial T 1041

Wohlfahrt J 1070

Wolf B 1276

Wolokoff B 635

Wong AJ 186

Wong J 538

Woods Ignatoski KM 666

Woszczynski M 1041

Wright DH 1117

Wu AH 1879

Wu C-H 1035

Wyllie AH 1247

Wyshak G 726

Xia X-M 683

Xian CJ 945

Yagi K 28

Yajima T 1717

Yamada T 1945

Yamada Y 467

Yamamoto H 1677

Yamamoto K 404, 675, 833

Yamamoto S 46, 418

Yamazaki M 1717
Yan PS 514

Yandell DW 579

Yang C-C 1480

Yang Q 763

Yang S-D 1480

Yap WM 1198

Yokosaki Y 1211

Yokota A 88

Yoo J-Y 683

Yoon K-A 1403

Yoshiga K 838

Yoshikawa Y 459

Yoshimi N 467

Young CYF 361

Young H 608

Young IE 1247

Yu J 1795

Yu MC 1364

Yuan F 1513

Yuan J-M 1364

Zagożdżon R 1485

Zambon M 126

Zambon P 227

Zarandi M 1724

Závada J 1808

Závadová Z 1808

Zeigler ME 657

Zekri J 858

Zhang C 1879

Zhou Y 459

Zielinski C 1249

Zielinski D 1421

Zimmerman PV 119

Zucchetti M 524 